\title{
Elastic Image Registration with Applications to Proteomics
}

\author{
C.O.S. Sorzano*, P. Thévenaz ${ }^{\dagger}$, I. Valdés**, A. Beloso** and M. Unser ${ }^{\dagger}$ \\ ${ }^{*}$ Escuela Politécnica Superior, Univ. San Pablo-CEU, Madrid, Spain. \\ ${ }^{\dagger}$ École Polytechnique Fédérale de Lausanne (EPFL), Switzerland. \\ ${ }^{*}$ Proteomic Lab., National Center of Biotechnology (CSIC), Madrid, Spain.
}

\begin{abstract}
In proteomics, electrophoretic 2-D gels are used to separate the proteome according to the molecular weight and electrical charge of its constituents, which are proteins expressed by a cell or organ at a particular time and under specific conditions. One of the main applications is the analysis of differential expressions between different conditions for which certain (perhaps many) spots are present in one of the images, but not in the other. One of the difficulties of this analysis is that 2-D gels are affected by spatial distortions due to run-time differences and dye-front deformations, which results in images that are significantly dissimilar and that pose a challenging problem to image-registration algorithms. In this paper, we test the efficiency of a stateof-the-art elastic-registration algorithm that we had already introduced in the context of biomedical images [1]. We study here the registration of simulated 2-D gels with known expression patterns and deformations. We show that our algorithm is capable of handling such situations. The proposed algorithm is publicly available at http://bigwww.epfl.ch under the name UnWarpJ.
\end{abstract}

Keywords: Image registration, 2D gels, B-splines

PACS: $87.57 . \mathrm{Gg}$

\section{INTRODUCTION}

2-D electrophoresis is a procedure to separate and identify the proteins expressed by an organ, tissue, or cell, at a given time and under certain conditions [2]. A complex mixture of proteins is injected into a polyacrylamide gel. Then, proteins are first separated according to their electric charge by a $\mathrm{pH}$ gradient and, then, according to their mass by electrophoresis in the presence of SDS. Finally, a dye is applied with a selective affinity toward specific components of the proteins. The result is a bidimensional image where small spots reveal the presence of a protein with a certain mass and charge in the initial solution (see Fig. 1).

One of the main applications of 2-D electrophoresis is the detection of differential expression, which is characterized by variations in the patterns of proteins an organism expresses under different situations, or by the different patterns expressed by different strains of organisms. The analysis of differential expression involves a careful registration between two gels.

Putting two images into registration can be restated as finding a function (also called a deformation field) that can be used to map a source image onto a target image [3]. In the particular case of electrophoretic 2-D gels, the registration poses some challenging obstacles that need to be overcome. In most cases, the deformation differs markedly from a simple rigid-body or affine transformation. Instead, electrophoresis images usually

CP860, Information Optics: $5^{\text {th }}$ International Workshop, edited by G. Cristóbal, B. Javidi, and S. Vallmitjana

(C) 2006 American Institute of Physics 978-0-7354-0356-7/06/\$23.00 


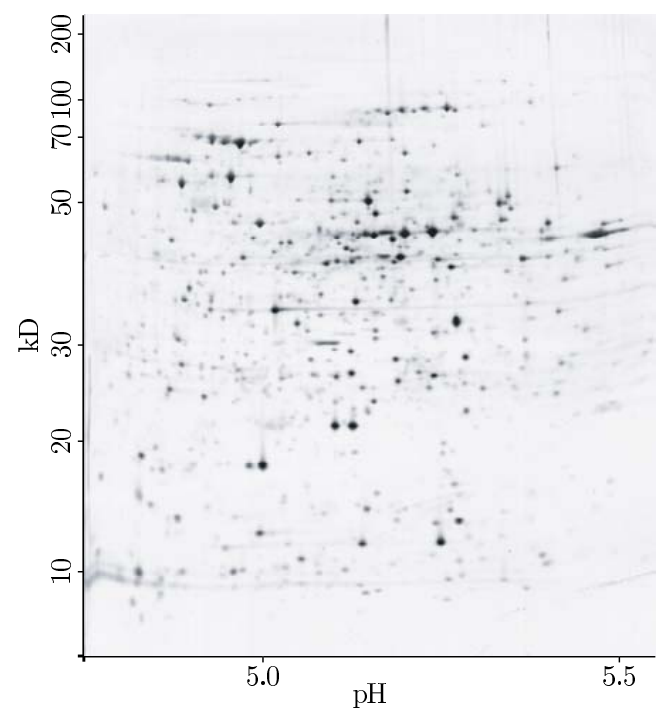

FIGURE 1. Gel example corresponding to Escherichia coli from the Swiss-2DPage database (entry: http://www.expasy.org/ch2dgifs/ECOLI4.5-5.5/ECOLI4.5-5.5.gif). The horizontal axis corresponds to the electrical charge and the vertical axis corresponds to the molecular weight.

require local and non-linear deformations, which only an elastic-deformation framework can handle properly. As additional difficulty, the information content of the images is most of the times distributed in uneven fashion (e.g., there might be no relevant information outside restricted image patches). Finally, it is inherently impossible to register perfectly the two images since it is their difference that carries the useful information.

Most of the image-registration algorithms (or "matching", as referred to in electrophoresis) involve pairs of spots that either are detected automatically, or that need to be explicitly provided by the user [4]. To the best of our knowledge, the only registration methods that work directly on the electrophoretic image information can be found in $[5,6,7,8]$. The method in [5] solves a differential equation for the transformation field after smartly deriving an image formation model. The method in [6] can also handle landmarks (known spot pairs), image intensities and a regularization term. The elastic transformation field is not parametrized and the regularization term is based on its quadratic energy. The methods in $[7,8]$ produce a piecewise-bilinear mapping. In [7], the grid is generated by a Delaunay triangulation, while a regular grid is deformed to adapt to the image characteristics in [8]. None of these two algorithms benefits from landmarks or from regularization in the optimization process, a limitation that we overcome with the algorithm presented in this paper.

In [1] we proposed the combined use of three components to correct for the deformations that one usually encounters in 2-D gels: the image content, spot pairs, and regularization. The image content has been shown to provide enough information as to drastically decrease the amount of spot pairs needed to achieve a given registration 
acccuracy. However, spot pairs can still be integrated so that the algorithm can benefit from user knowledge. The use of spot pairs is particularly useful to compensate for the information mismatch between images (spots that appear in only one image) and the uneven distribution of the information (large areas without spots). Furthermore, the image matching may be restricted to a user-specified region. This possibility is also very useful for avoiding large empty or mismatching regions. We also proposed the use of B-splines to represent the deformation field as well as the images. In this way, nonlinear elastic deformation models can be produced [9] and a high-quality interpolation is available [10]. The deformation field is estimated through a minimization problem that includes the energy of the error between both images, the error in the mapping of corresponding spots, and a smoothness regularization term. We proposed to use a fully vectorial regularization term based on vector splines [11]. This approach is a generalization of more usual regularization terms in 2D and provides extra freedom that is particularly useful to correct certain typical effects in gels like the dye-front deformation [12]. The optimizer used is a Levenberg-Marquardt-like algorithm already proposed by Thévenaz et al. [13] and enhanced by a BFGS (Broyden-Fletcher-Goldfarb-Shanno) estimation of the Hessian [14]. The optimization is performed in a multiresolution fashion to increase the robustness and speed of the algorithm. The exact analytical solution is provided in the case that the data term is neglected.

In this paper, we test the applicability of our image-registration algorithm to the analysis of differential gels under different run times and protein expression patterns using simulated data.

\section{MATERIALS AND METHODS}

We select B-splines to model the images, which ensures their high-quality interpolation. Similarly, we also use B-splines to model the deformation field. In this way, a nonlinear elastic-deformation model can be produced while keeping a tight control on its level of detail. This deformation field is estimated through a minimization problem that includes three terms: the measure of dissimilarity $E_{\text {img }}$ between the target image and the warped source image, the soft-landmark constraints $E_{\mu}$, and a priori knowledge about the deformation field through the two independent measures $E_{\text {div }}$ and $E_{\text {rot }}$ that are related to the gradients of divergence and curl of the vectorial deformation field, respectively. Our algorithm is also capable of making use of additional a priori knowledge such as masks and landmarks. Thus, the energy to minimize is a linear combination of these energy terms, as in

$$
E=w_{i} E_{\mathrm{img}}+w_{\mu} E_{\mu}+\left(w_{d} E_{\mathrm{div}}+w_{r} E_{\mathrm{rot}}\right) .
$$

We suggest to assist the efficient optimization method of Levenberg and Marquardt by the accurate Broyden-Fletcher-Goldfarb-Shanno (BFGS) estimate of the Hessian. Moreover, the optimization process is performed in a multiresolution framework in which coarse representations of the source and target images are coarsely registered. Then, finer details in the images and finer registration fields are alternatively computed until reaching the desired level of accuracy. This approach has proved to be helpful for avoiding local minima in other biomedical imaging applications. The different degrees 
of accuracy in the image representation (coarse, fine, finer, etc.) yield a pyramid of images whose resolution is better as they get closer to the pyramid base. The number of images in the stack is referred to as the image pyramid depth. Similarly, the different deformation field levels yield the deformation pyramid.

\section{Data Term}

The goal of image registration is to find a function $\mathbf{g}(\mathbf{x}): \mathbb{R}^{2} \rightarrow \mathbb{R}^{2}$ so that the warped version $I_{s}(\mathbf{g}(\mathbf{x}))$ of the source image $I_{s}$ resembles the target image $I_{t}$ as much as possible. Moreover, when masks are considered, we ought to concentrate our attention to regions of interest within the source and target images. Let us call $\Omega_{s} \subset \mathbb{R}^{2}$ and $\Omega_{t} \subset \mathbb{Z}^{2}$ the regions of interest of the source and target images, respectively. Then, we measure the dissimilarity between the warped source image and the target image by the discrete sum

$$
E_{\mathrm{img}}=\frac{1}{\# \Omega} \sum_{\mathbf{k} \in \Omega}\left(I_{t}(\mathbf{k})-I_{S}(\mathbf{g}(\mathbf{k}))\right)^{2},
$$

where $\Omega$ defines a mask common to the source and target images, and where $\# \Omega$ is the number of pixels of this mask.

\section{Deformation Representation}

We express the deformation field as the linear combination of B-splines given by

$$
\begin{aligned}
\mathbf{g}(\mathbf{x}) & =\mathbf{g}(x, y) \\
& =\left(g_{1}(x, y), g_{2}(x, y)\right) \\
& =\sum_{k, l \in \mathbb{Z}^{2}}\left(\begin{array}{l}
c_{1, k, l} \\
c_{2, k, l}
\end{array}\right) \beta^{3}\left(\frac{x}{s_{x}}-k\right) \beta^{3}\left(\frac{y}{s_{y}}-l\right),
\end{aligned}
$$

where $s_{x}$ and $s_{y}$ are scalars that control the degree of detail of the representation of the deformation field. Cubic B-splines constitute a Riesz basis of $L_{2}$ and have a fourth order of approximation. Therefore, any sufficiently regular deformation field can be represented with vanishing error using a fine-enough scale. Moreover, by using B-splines of degree 3, the continuity of the derivatives of the deformation is guaranteed up to the second order, which is favorable to optimization schemes based on derivatives. Another advantage of this model is that any affine transformation (rotation, shifting, scaling or any combination of them) can be represented by our B-spline deformation.

\section{Spot pairs}

We benefit from spot pairs in two different ways: first, we use spots to impose soft constraints to the deformation field; second, we use spots during the initialization of the 
optimization problem. Let us assume that $N$ pairs of corresponding spots $\left(\mu_{s}^{(n)}, \mu_{t}^{(n)}\right)$, $n \in\{1 \ldots N\}$, are available. Then, we handle the soft-landmark constraints by an energy term of the form

$$
E_{\mu}=\frac{1}{N} \sum_{n=1}^{N}\left\|\mu_{t}^{(n)}-\mathbf{g}\left(\mu_{s}^{(n)}\right)\right\|^{2} .
$$

\section{Regularization}

The smoothness of the deformation field is a useful regularization term for the minimization problem, especially when little information is available. Considering that $\mathbf{g}$ is a 2-D vector field, we impose smoothness to this field by the following two regularization terms that fully exploit the vectorial nature of the data:

$$
\begin{aligned}
E_{\mathrm{rough}}= & w_{d} \int_{\mathbb{R}^{2}}\|\nabla \operatorname{div}(\mathbf{g}(\mathbf{x}))\|^{2} \mathrm{~d} x \mathrm{~d} y \\
& +w_{r} \int_{\mathbb{R}^{2}}\|\nabla \operatorname{rot}(\mathbf{g}(\mathbf{x}))\|^{2} \mathrm{~d} x \mathrm{~d} y,
\end{aligned}
$$

where $\operatorname{div}(\mathbf{g})$ represents the divergence of the 2-D vector field $\mathbf{g}$, where $\operatorname{rot}(\mathbf{g})$ represents the length of the unique component of the curl of $\mathbf{g}$, and where $\nabla=\left(\partial_{x}, \partial_{y}\right)$ is the bidimensional gradient operator.

An important advantage of using this regularization structure is that it gives the user more freedom to express her a priori knowledge about the underlying deformation. In particular, the "smile" effect can be easily corrected by giving a heavy weight to the rotational-based regularization term, as shown in [1].

\section{RESULTS}

To perform objective tests of the efficiency of our algorithm, we have applied our registration algorithm to three different synthetic datasets so that we could have a total control over how they would differ. Our goals with these datasets are to evaluate the possibility of correcting for dye-front deformations, to evaluate the amount of information provided by each of the goal function terms (data, spots, regularization), and to assess the algorithm performance under some realistic situations.

\section{Dataset 1: Synthetic data simulating dye-front deformations}

A common deformation in gel electrophoresis is known as the "smile effect" (dyefront deformation, [12]). It can be proved [1] that this distortion belongs to a class of deformations whose divergence gradient is null. This means that we can tell our algorithm to penalize deformation fields with high changes in their divergence (since the sought solution has in fact none). This is, in principle, advantageous versus other image registration algorithms since we can easily control the set from which the deformation field will be picked. 

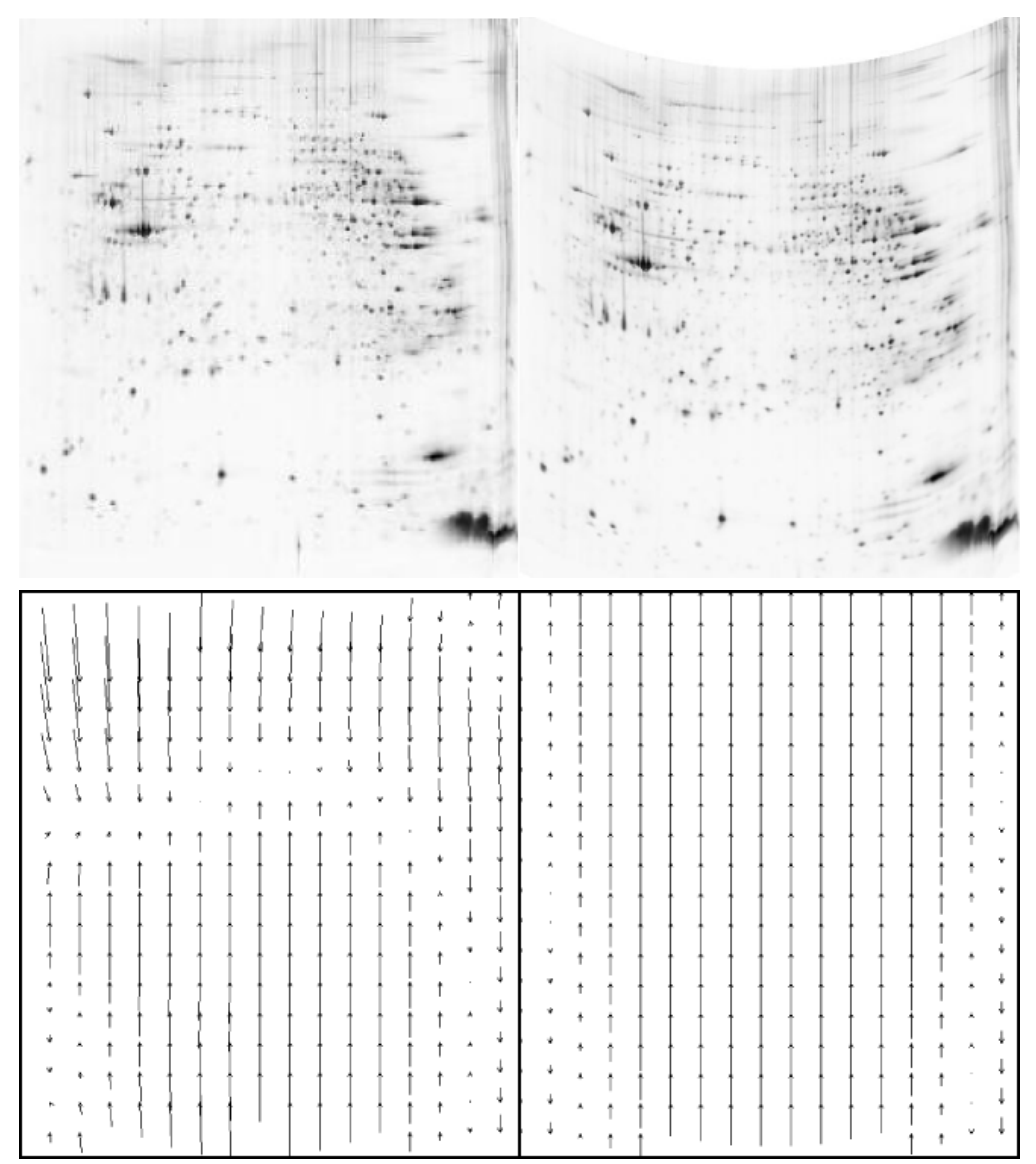

FIGURE 2. Top: Pair of corresponding 2D gels related by a "smile" transformation. The image on the left is unprocessed data, while the image on the right was simulated by Raman et al. [4]. Bottom left: Deformation field of the unregularized registration. Bottom right: Deformation field of the regularized registration.

For these tests we downloaded the data used by [4] in their article comparing several commercial solutions to the image matching problem from http:// www.umbc.edu/proteome. We give in Figure 2 an example of this deformation with a factor reduction of $5 \%$ and a maximum central shift of $14 \%$. This image is compared to its undeformed version and the resulting deformation field is computed. The unregularized problem converges to an unsatisfactory solution as can be seen in Figure 2. The problem is that the resulting deformation field is not sufficiently regular (it is known that the deformation field has no horizontal component, i.e., all arrows should be vertical and that they should not change their directions). However, successful unwarping is achieved with $w_{d}=4$ and $w_{r}=0$ (with $w_{i}=1$ and $w_{\mu}=0$ ). The deformation pyramid had 2 levels, and the image pyramid had 3 levels. 


\section{Dataset 2: Synthetic data to evaluate the influence of each goal function term}

Most of the works in electrophoresis image registration is based on spot matching [15, $16,17,18,19,20]$. On one hand, matching spots is a more complex problem than matching landmarks since their correspondence is unknown and since the number of spots in both images may differ. On the other hand, the spot extraction can be done fully automatically while landmarks require user input.

The effect of using the image data in our algorithm is two-fold: first, the registration accuracy is greatly improved; second, the convergence to the final solution is substantially accelerated. The number of landmarks needed to achieve the right solution ranges from 0 to about 10 depending on the complexity of the underlying deformation field and the amount of spots available in the gel [1].

Although our algorithm is capable of combining landmarks and image data, some approaches work with landmarks only. In the next experiment, we adjust the number of perfect landmarks necessary to achieve about the same registration accuracy as when the data term is included in the minimization problem. We generated thirty random "smile" deformations. The reduction factor was uniformly distributed between $0 \%$ and $5 \%$, while the maximum central shift was uniformly distributed between $0 \%$ and $15 \%$. The deformation field was always represented with $4 \times 4$ grid points.

For the experiment taking into account the content of the images, we put a random landmark in each of the four image quadrants. To simulate the uncertainty on the landmarks when they are manually selected, we added random Gaussian noise with zero mean and standard deviation 1 pixel to the $x$ and $y$ components of each of these four landmarks. The deformation field had two pyramid levels, and the image pyramid three. The weights of the data term and the landmarks were both set to $w_{i}=w_{\mu}=1$, while the weight of the regularizing term based on the divergence was set to $w_{d}=4$. We show the achieved accuracy in the first line of Table 1 . The accuracy of this experiment is computed using the warping index, a standard measure of the misregistration between two images [21, 1, 13]. This Figure of Merit measures the difference in pixel units between the right position of a certain point of the image and the position assigned by the algorithm. The initial warping index for this experiment was $20.52 \pm 12.80$.

For the landmark-only experiments (remaining lines of Table 1), we generated $N$ random landmarks. The first four landmarks were distributed as described for the previous experiment, while the rest of the landmarks were randomly distributed all over the image. No noise was added to the landmark positions, assuming that the automatic landmark-selection algorithm performed a perfect job. We conducted two experiments: one with regularization $\left(w_{\text {div }}=4, w_{\text {rot }}=0\right)$, and another one without regularization, discounting the implicit regularization present in the $4 \times 4$ B-spline-based deformation model $\left(w_{\text {div }}=w_{\text {rot }}=0\right)$. We show in Table 1 the achieved accuracy and the number of landmarks needed so that the hypothesis that the average final warping index is the same for the three experiments cannot be rejected with a confidence of $99 \%$. 
TABLE 1. Final warping index when the image information is considered $\left(w_{i} \leq 0\right)$ and the problem is regularized $\left(w_{d} \leq 0\right)$, when the image information is disregarded $\left(w_{i}=0\right)$ but the problem is still regularized, and when both image data and regularization are ignored (landmark-only, $w_{i}=w_{d}=w_{r}=0$ ). In each case, $N$ landmarks are always considered $\left(w_{\mu}=\right.$ $0)$.

\begin{tabular}{r|r|r|r|r|l}
\hline \hline$w_{i}$ & $w_{\mu}$ & $w_{d}$ & $w_{r}$ & $N$ & Warping index \\
\hline 1 & 1 & 1 & 0 & 4 & $0.060 \pm 0.059$ \\
0 & 1 & 4 & 0 & 90 & $0.090 \pm 0.056$ \\
0 & 1 & 0 & 0 & 190 & $0.099 \pm 0.070$ \\
\hline \hline
\end{tabular}

TABLE 2. Parameters used to synthesize gels.

\begin{tabular}{c|c|c|c|c}
\hline \hline Image name & Strain & \#Proteins & Run time & Smile \\
\hline$I_{1}$ & EDL933 & 4441 & 10 & No \\
$I_{2}$ & EDL933 & 3069 & 10 & No \\
$I_{3}$ & EDL933 & 3417 & 20 & No \\
$I_{4}$ & EDL933 & 2395 & 10 & Yes \\
$I_{5}$ & KL12 & 3734 & 10 & No \\
\hline
\end{tabular}

\section{Dataset 3: Synthetic data simulating realistic conditions}

Thanks to the JVirgel program [22], we were able to simulate 2-D gels for the proteomes of the Escherichia coli strains EDL933 [23] and K12 [24]. We have simulated five different gels by varying parameters such as run time, strain, expression pattern, and dye deformation. We show in Table 2 the different combinations that we have explored in this paper. The run time represents the time needed to drive proteins of 10 or $20 \mathrm{kD}$ down to the bottom of the gel area. The number of proteins within the gel depends on the run time (higher run times imply fewer proteins within the gel limits). To generate images $I_{2}$ and $I_{4}$, we have arbitrarily removed all those proteins whose name starts with any letter in the alphabetic range "a" to " $h$ " (inclusive) to simulate a differential expression pattern between two different conditions. Since the images generated by JVirgel are binary, we have blurred them with a Gaussian filter in order to better simulate real data. The standard deviation of this filter was 1 pixel.

We have performed four different experiments, with the goal of showing each time a different aspect of the registration problem that one may actually encounter in real data. These experiments are as follows:

- $I_{1}$ vs. $I_{2}:$ an important $(\approx 31 \%)$ fraction of the proteins are inhibited (different expression pattern).

- $I_{1}$ vs. $I_{3}$ : different run times. This is one of the most difficult aspect to control in the repeatability of gels. 
- $I_{1}$ vs. $I_{4}$ : different expression patterns combined with a dye-front distortion.

- $I_{1}$ vs. $I_{5}$ : two different strains of the same organism.

We never provided landmarks to any of the experiments, except in the $I_{1} v s . I_{3}$ case, due to the huge difference between these two images. There, we selected three landmarks and we used them only for initialization. We set the parameters of the algorithm to $w_{i}=w_{r}=1$ and $w_{\mu}=w_{d}=0$. We show the results of the four experiments in Figure 3 .

\section{ACKNOWLEDGMENTS}

This work has been partially supported by "Comunidad de Madrid" through grant GR/SAL/0342/2004 and by the Swiss National Science Foundation through grant 2153066938.

\section{REFERENCES}

1. C. O. S. Sorzano, P. Thévenaz, and M. Unser, IEEE Trans. on Biomedical Eng. 52, 652-663 (2005).

2. A. Gorg, W. Weiss, and M. Dunn, Proteomics 4, 3665-3685 (2004).

3. B. Zitova, and J. Flusser, Image and Vision Computing 21, 977-1000 (2003).

4. B. Raman, A. Cheung, and M. R. Marten, Electrophoresis 23, 2194-2202 (2002).

5. J. S. Gustafsson, A. Blomberg, and M. Rudemo, Electrophoresis 23, 1731-1744 (2002).

6. K. Rohr, P. Cathier, and S. Worz, Pattern recognition 37, 1035-1048 (2004).

7. Z. Smilansky, Electrophoresis 22, 1616-1626 (2001).

8. S. Veeser, M. J. Dunn, and G. Z. Yang, Proteomics 1, 856-870 (2001).

9. R. Szeliski, and J. Coughlan, Intl. J. Computer Vision 22, 199-218 (1997).

10. M. Unser, A. Aldroubi, and M. Eden, IEEE Trans. Pattern Analysis \& Machine Intelligence 13, 277-285 (1991).

11. L. Amodei, and M. N. Benbourhim, J. of Approximation Theory 67, 51-79 (1991).

12. Biorad Laboratories, Mini Protean 3: Electrophoresis instruction manual (2003).

13. P. Thévenaz, U. E. Ruttiman, and M. Unser, IEEE Trans. Image Processing 7, $27-41$ (1998).

14. W. Press, S. A. Teukolsky, W. T. Vetterling, and B. P. Flannery, Numerical recipes in C, Cambridge University Press, 1992, 2 edn.

15. T. Akutsu, K. Kanaya, A. Ohyama, and A. Fujiyama, Discrete Applied Mathematics 127, 5-21 (2003).

16. J. Panek, and J. Vohradsky, Electrophoresis 20, 3483-3491 (1999).

17. R. D. Appel, J. R. Vargas, P. M. Palagi, D. Walther, and D. F. Hochstrasser, Electrophoresis 18, 2735-2748 (1997).

18. K. Kriegel, I. Seefeldt, F. Hoffmann, C. Schultz, C. Wenk, V. Regitz-Zagrosek, H. Oswald, and E. Fleck, Electrophoresis 21, 2637-2640 (2000).

19. P. F. Lemkin, Electrophoresis 18, 461-470 (1997).

20. J. Salmi, T. Aittokallio, J. Westerholm, M. Griese, A. Rosengren, T. A. Nyman, R. Lahesmaa, and O. Nevalainen, Proteomics 2, 1504-1515 (2002).

21. J. Kybic, and M. Unser, IEEE Trans. Image Processing 12, 1427-1442 (2003).

22. K. Hiller, M. Schobert, C. Hundertmark, D. Jahn, and R. Münch, Nucleic Acids Research 31, 3862 3865 (2003).

23. V. Burland, Y. Shao, N. Perna, G. I. Plunkett, H. Sofia, and F. Blattner, Nucleic Acids Research 26, 4196-4204 (1998).

24. F. Blattner, G. I. Plunkett, C. Bloch, N. Perna, V. Burland, M. Riley, J. Collado-Vides, J. Glasner, C. Rode, G. Mayhew, J. Gregor, N. Davis, H. Kirkpatrick, M. Goeden, D. Rose, B. Mau, and Y. Shao, Science 277, 1453-1474 (1997). 

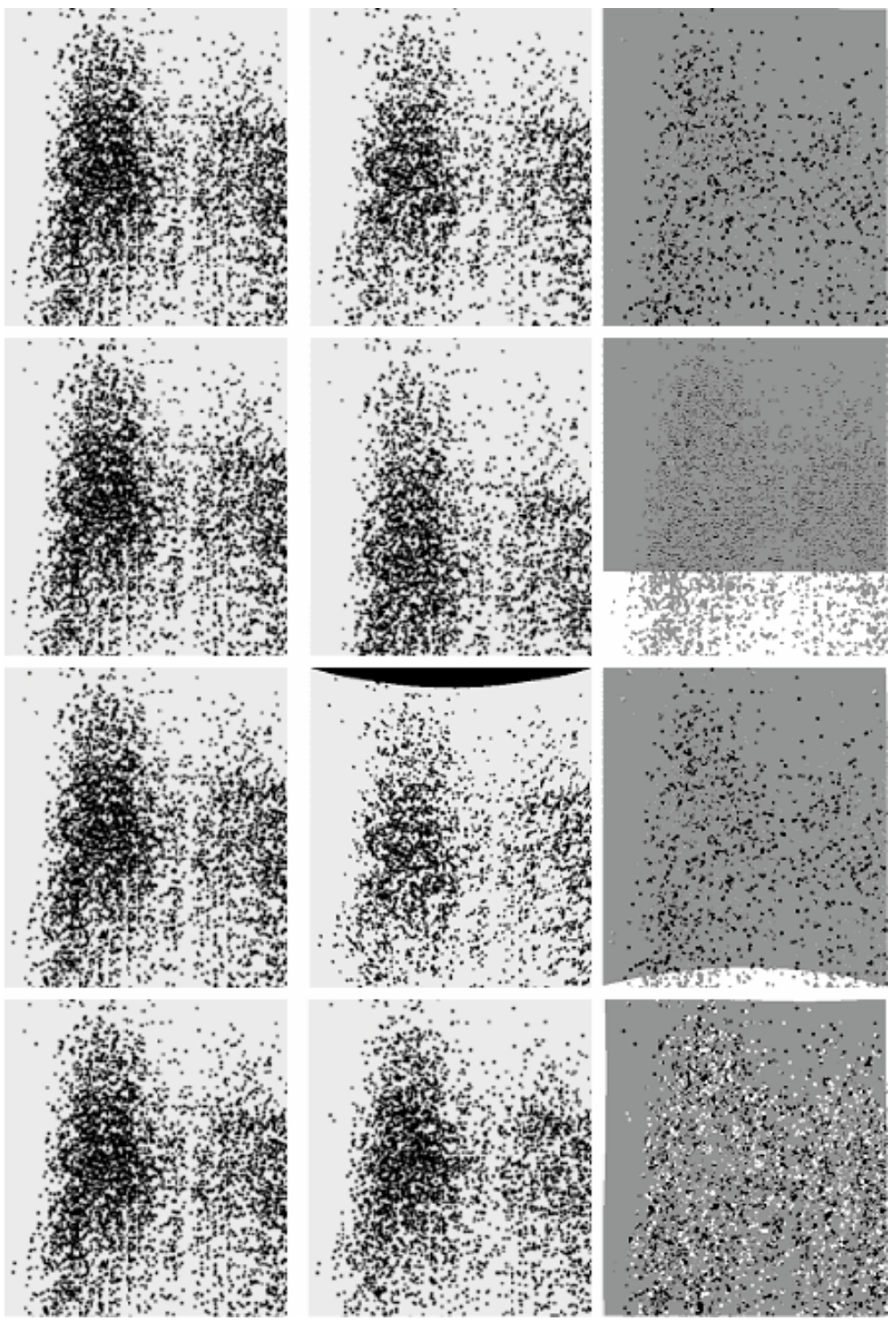

FIGURE 3. Each row represents a different experiment. From top to bottom: $I_{1} v s . I_{2}, I_{1} v s . I_{3}, I_{1} v s$. $I_{4}$, and $I_{1} v s . I_{5}$. For each experiment, we show the target image $I_{1}$, the source image $I_{i}$, and the difference $\left(I_{i}-I_{1}\right)$ between the registered image (warped source) and the target image. 